\title{
Velocity correlations in liquid hydrogen fluoride
}

U. Balucani, D. Bertolini, G. Sutmann, A. Tani, and R. Vallauri

Citation: The Journal of Chemical Physics 111, 4663 (1999); doi: 10.1063/1.479228

View online: https://doi.org/10.1063/1.479228

View Table of Contents: http://aip.scitation.org/toc/jcp/111/10

Published by the American Institute of Physics

\section{Articles you may be interested in}

Comparison of simple potential functions for simulating liquid water

The Journal of Chemical Physics 79, 926 (1983); 10.1063/1.445869

Analysis of the hydrogen-bonded structure of water from ambient to supercritical conditions

The Journal of Chemical Physics 108, 8528 (1998); 10.1063/1.476282

Molecular Dynamics Study of Temperature Effects on Water Structure and Kinetics

The Journal of Chemical Physics 57, 1281 (1972); 10.1063/1.1678388

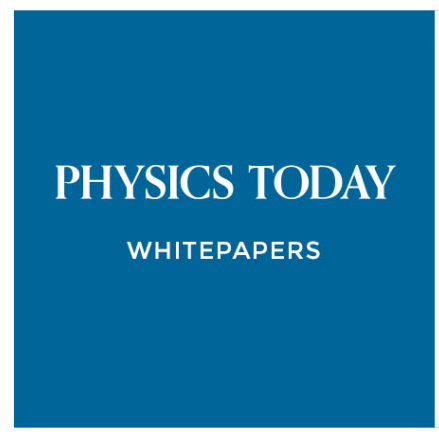




\title{
Velocity correlations in liquid hydrogen fluoride
}

\author{
U. Balucani \\ Istituto di Elettronica Quantistica del Consiglio Nazionale delle Ricerche, I-50127 Firenze, Italy \\ D. Bertolini \\ Istituto di Fisica Atomica e Molecolare del Consiglio Nazionale delle Ricerche, I-56100 Pisa, Italy
}

G. Sutmann

Research Center Jülich, Central Institute for Applied Mathematics, D-52425 Jülich, Germany

A. Tani

Dipartimento di Chimica e Chimica Industriale, Università di Pisa, I-50126 Pisa, Italy

R. Vallauria)

Istituto Nazionale di Fisica della Materia and Dipartimento di Fisica, Università di Trento, via Sommarive 14, I-38050 Povo (Trento), Italy

(Received 5 April 1999; accepted 16 June 1999)

The center-of-mass velocity autocorrelation function is analyzed by computer simulation in a model of liquid hydrogen fluoride at two state points. In comparison with water (another hydrogen-bonded liquid) new features arise. To understand the peculiarities of HF, we have investigated atomic velocity correlations in both the laboratory and a molecular frame. The comparison of the frequency spectra permits to ascertain the role of fluorine-hydrogen correlations (or of rototranslational couplings) in the center-of-mass velocity autocorrelation function. At low temperature, the appearance of a long time tail is discussed in terms of projections in the two references frames, and found to be mostly associated with orientational correlations. A discussion in terms of velocity transfer between nearest-neighbor molecules is also given. (C) 1999 American Institute of Physics. [S0021-9606(99)52034-5]

\section{INTRODUCTION}

Among hydrogen-bonded systems liquid water is undoubtedly the most important because of its role in chemical and biological processes, and therefore has widely been investigated from both the experimental and the theoretical point of view. A basic subject of investigation is to assess the relevance of the hydrogen bond on those features absent in nonassociated liquids; for this purpose, a comparative study of water and other hydrogen-bonded liquids is clearly desirable.

In the present investigation we shall deal with liquid hydrogen fluoride, known as the system with the strongest hydrogen bond. Because of its high reactivity, the structural and dynamical properties of HF are much less known than those of water; however, the ultimate factor in the establishment of the hydrogen bond is similar in the two cases, being basically determined by strong electrostatic forces. As a result, $\mathrm{HF}$ and $\mathrm{H}_{2} \mathrm{O}$ do indeed share some aspects in their microscopic structure; in both systems, the two heavy particles can approach each other closer than expected by their "size," a circumstance which in turn leads to unusually high values of the Einstein frequency $\Omega_{0}$. These common features must, however, be contrasted with even more important differences. While in water the molecules are arranged in a nearly tetrahedral three-dimensional network (with oxy-

\footnotetext{
a) Author to whom correspondence should be addressed. Electronic mail: vallauri@science.unitn.it
}

gen coordination number $\approx 4$ ), liquid $\mathrm{HF}$ instead consists of long irregular chains with fluorine coordination number $\approx 2{ }^{2}$ These structural differences have an immediate counterpart in the dynamical properties of the two fluids. In many respects, the molecular motions in HF can be considered as "more free" than in water; in particular, when compared in suitable reduced units transport properties such as the diffusion coefficient $D$ and the fluidity $1 / \eta$ are considerably larger in $\mathrm{HF}$ than in $\mathrm{H}_{2} \mathrm{O} .{ }^{3}$ Moreover, a peculiar phenomenon occurring in the collective longitudinal dynamics of water (the so-called "fast sound",4) is nearly absent in HF, and welldefined shear excitations at non hydrodynamic wave vectors are much more easily detected in $\mathrm{H}_{2} \mathrm{O}$ than in $\mathrm{HF}^{5}{ }^{5}$

As far as microdynamics is concerned, the above phenomena suggest the presence in the two systems of considerably different relaxation mechanisms. It is easily anticipated that this diversity is not confined to collective dynamics but reflects a distinct behavior at the basic level of single-molecule time correlation functions. ${ }^{6}$ In this respect, the simplest "self" dynamical quantity is the velocity autocorrelation function (VACF) of the molecular centers of mass. When compared with the evolution of the same quantity in a realistic model of liquid water (Fig. 1), several basic differences are immediately apparent in the VACF of HF at low temperature; (i) at short and intermediate times much more pronounced oscillations are present, and (ii) at long times the VACF of HF at $203 \mathrm{~K}$ shows a "tail" of considerable duration which is virtually absent in water (although we have purposely chosen a nominal "supercooled" state at 


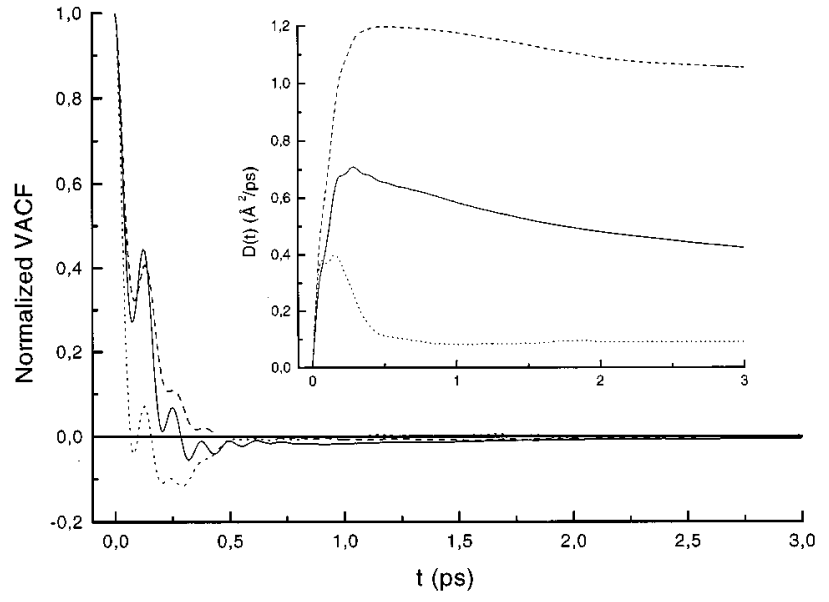

FIG. 1. Normalized center-of-mass velocity autocorrelation function (VACF) for liquid $\mathrm{HF}$ at $203 \mathrm{~K}$ (full line) and $273 \mathrm{~K}$ (dashed line). The data are compared with those obtained for the SPC/E model of "supercooled" water at $255 \mathrm{~K}$ (dotted line). The insert shows the quantity $D(t)$ $=(1 / 3) \int_{0}^{t} d \tau C_{c}(\tau)$ for the three VACFs up to $t=3 \mathrm{ps}$.

$255 \mathrm{~K})$ as well as in a high-temperature realization of HF. The presence of these long-lasting tails makes rather demanding an accurate evaluation of the diffusion coefficient of HF at the lowest temperature (Fig. 2), and consequently is important even at a macroscopic level.

The main goal of this work is to understand these findings by a thorough investigation of the dynamical processes occurring in liquid HF. In Sec. II we report the definitions of the dynamical quantities to be explored as well as an outline of our molecular dynamics (MD) simulation. Section III deals with a closer examination of single particle motion in $\mathrm{HF}$ as provided by MD data for the partial velocity correlation functions $(\mathrm{F}, \mathrm{F}),(\mathrm{H}, \mathrm{H})$, and $(\mathrm{F}, \mathrm{H})$. Moreover, by an alternative analysis it is possible to investigate the relevance of the coupling between translational and rotational motions in various time (or frequency) domains. We shall also find convenient to analyze the data obtained both in the fixed laboratory frame and in one associated with the rotating molecular axis. Having established this framework, Sec. IV is

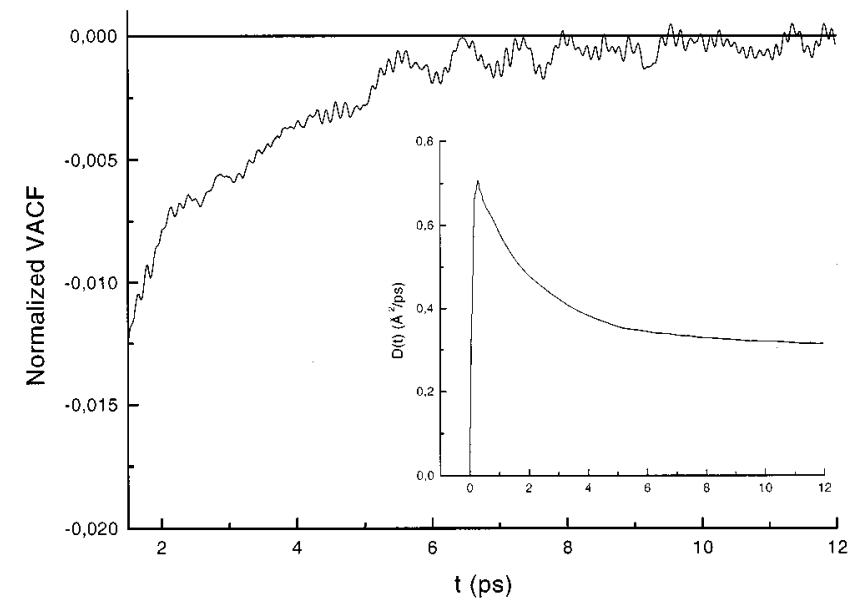

FIG. 2. Long-time behavior of the normalized VACF for liquid HF at 203 $\mathrm{K}$. The insert shows that a reliable determination of the diffusion coefficient $D$ requires the knowledge of the $\mathrm{VACF}$ at times as long as $\approx 10 \mathrm{ps}$. devoted to the discussion of both short- and long-time phenomena as reflected in the high- and low-frequency regions of the corresponding spectra. The content and conclusions of the paper are summarized in Sec. V. Some detailed results are reported in Appendices A and B.

\section{THE RELEVANT QUANTITIES AND THE MOLECULAR DYNAMICS SIMULATION}

In a system made by $N$ " $p$-atomic" molecules, we shall denote by $\mathbf{r}_{j}^{(\alpha)}(t)$ the position vector of the $\alpha$ th atom ( $\alpha$ $=1, \ldots, p)$ within the $j$ th molecule at time $t$. The corresponding velocity reads $\mathbf{v}_{j}^{(\alpha)}(t)=d \mathbf{r}_{j}^{(\alpha)}(t) / d t$. If $m_{\alpha}$ is the mass of the $\alpha$ th atom in the molecule and $M=\Sigma_{\alpha} m_{\alpha}$ the molecular mass, the position vector $\mathbf{r}_{j}^{(c)}$, and the velocity $\mathbf{v}_{j}^{(c)}$ of the center-of-mass of the $j$ th molecule at time $t$ follow from

$$
\begin{aligned}
& \mathbf{r}_{j}^{(c)}(t)=(1 / M) \sum_{\alpha} m_{\alpha} \mathbf{r}_{j}^{(\alpha)}(t), \\
& \mathbf{v}_{j}^{(c)}(t)=(1 / M) \sum_{\alpha} m_{\alpha} \mathbf{v}_{j}^{(\alpha)}(t) .
\end{aligned}
$$

Assuming that each molecule can be considered as rigid, the velocity $\mathbf{v}_{j}^{(\alpha)}(t)$ can be split into a purely translational component $\mathbf{v}_{j}^{(c)}(t)$ and a rotational component involving the angular velocity $\boldsymbol{\omega}_{j}(t)$ around the center-of-mass,

$$
\mathbf{v}_{j}^{(\alpha)}(t)=\mathbf{v}_{j}^{(c)}(t)+\boldsymbol{\omega}_{j}(t) \times \boldsymbol{\rho}_{j}^{(\alpha)}(t),
$$

where $\boldsymbol{\rho}_{j}^{(\alpha)}(t)=\mathbf{r}_{j}^{(\alpha)}(t)-\mathbf{r}_{j}^{(c)}(t)$. In the following we shall deal with single-molecule time correlation functions (CFs), and henceforth omit the molecular label $j$. Generally speaking, these CFs will be written as $C_{A B}^{(L)}(t)=\langle\mathbf{A}(0) \cdot \mathbf{B}(t)\rangle$, where the vectors $\mathbf{A}$ and $\mathbf{B}$ are associated with the velocities of the atoms in the molecule, and the dot indicates a scalar product in the usual laboratory $(L)$ frame spanned by the unit vectors $\mathbf{i}_{s}(s=x, y, z)$. In the following, it will be convenient to introduce even a mobile reference frame spanned by the time-dependent unit vectors $\mathbf{i}_{\sigma}(t)(\sigma=\xi, \eta, \zeta)$ in which the molecule under consideration appears as fixed. At time $t$ an arbitrary dynamical vector $\mathbf{G}$ can equivalently be expressed either as $\mathbf{G}(t)=\sum_{s} G_{s}(t) \mathbf{i}_{s}$, or as $\mathbf{G}(t)=\sum_{\sigma} G_{\sigma}(t) \mathbf{i}_{\sigma}(t)$. It is readily seen that

$$
\begin{aligned}
C_{A B}^{(L)}(t) & =\langle\mathbf{A}(0) \cdot \mathbf{B}(t)\rangle \\
& =\sum_{s}\left\langle A_{s}(0) B_{s}(t)\right\rangle \\
& =\sum_{\sigma}\left\langle\mathbf{A}(0) \cdot \mathbf{i}_{\sigma}(0) \mathbf{B}(t) \cdot \mathbf{i}_{\sigma}(0)\right\rangle .
\end{aligned}
$$

Let us now consider the quantity

$$
\begin{aligned}
C_{A B}^{(M)}(t) & \equiv \sum_{\sigma}\left\langle A_{\sigma}(0) B_{\sigma}(t)\right\rangle \\
& =\sum_{\sigma}\left\langle\mathbf{A}(0) \cdot \mathbf{i}_{\sigma}(0) \mathbf{B}(t) \cdot \mathbf{i}_{\sigma}(t)\right\rangle,
\end{aligned}
$$

which can naively be viewed as the time CF of a "scalar product in the molecular $(M)$ frame." Owing to molecular rotations, at $t \neq 0$ the quantity $\mathbf{i}_{\sigma}(0) \cdot \mathbf{i}_{\sigma^{\prime}}(t)$ is different from $\delta_{\sigma, \sigma^{\prime}}$. 
In the case of a diatomic molecule $(\alpha=1,2)$ the unit vector $\mathbf{i}_{\zeta}(t)$ can be chosen in the direction of the molecular axis at time $t$. With this choice, $\boldsymbol{\rho}^{(1)}(t)=-\rho_{1} \mathbf{i}_{\zeta}(t)$ and $\boldsymbol{\rho}^{(2)}(t)$ $=\rho_{2} \mathbf{i}_{\zeta}(t)$, where $\rho_{2}=\left(m_{1} / m_{2}\right) \rho_{1} \equiv r_{m} \rho_{1}$. The remaining unit vectors $\mathbf{i}_{\zeta}(t)$ and $\mathbf{i}_{\eta}(t)$ are arbitrary (see afterwards), but certainly lie in the plane perpendicular to the instantaneous molecular axis. The rigid motion relation (2) now takes the form

$$
\mathbf{v}^{(\alpha)}(t)=\mathbf{v}^{(c)}(t)+(-1)^{\alpha} \rho_{\alpha}\left|\boldsymbol{\omega}(t) \times \mathbf{i}_{\zeta}(t)\right| .
$$

In the following we shall consider the VACFs,

$$
\begin{aligned}
& C_{c}^{(L)}(t)=\left\langle\mathbf{v}^{(c)}(0) \cdot \mathbf{v}^{(c)}(t)\right\rangle, \\
& C_{\alpha \beta}^{(L)}(t)=\left\langle\mathbf{v}^{(\alpha)}(0) \cdot \mathbf{v}^{(\beta)}(t)\right\rangle,
\end{aligned}
$$

where $\alpha, \beta=1,2$. In the case of interest (HF) the labels 1 and 2 will denote the heavy (fluorine) and the light (hydrogen) atoms, respectively. The counterparts of Eqs. (6) and (7) in the $(M)$ frame read

$$
\begin{aligned}
C_{c}^{(M)}(t)= & \left\langle\mathbf{v}_{\zeta}^{(c)}(0) \mathbf{v}_{\zeta}^{(c)}(t)\right\rangle \\
& +\left[\left\langle\mathbf{v}_{\xi}^{(c)}(0) \mathbf{v}_{\xi}^{(c)}(t)\right\rangle+\left\langle\mathbf{v}_{\eta}^{(c)}(0) \mathbf{v}_{\eta}^{(c)}(t)\right\rangle\right] \\
\equiv & C_{c, \|}^{(M)}(t)+2 C_{c, \perp}^{(M)}(t), \\
C_{\alpha \beta}^{(M)}(t)= & \left\langle\mathbf{v}_{\zeta}^{(\alpha)}(0) \mathbf{v}_{\zeta}^{(\beta)}(t)\right\rangle \\
& +\left[\left\langle\mathbf{v}_{\xi}^{(\alpha)}(0) \mathbf{v}_{\xi}^{(\beta)}(t)\right\rangle+\left\langle\mathbf{v}_{\eta}^{(\alpha)}(0) \mathbf{v}_{\eta}^{(\beta)}(t)\right\rangle\right] \\
\equiv & C_{\alpha \beta, \|}^{(M)}(t)+2 C_{\alpha \beta, \perp}^{(M)}(t),
\end{aligned}
$$

where the symbols $\|$ and $\perp$ denote contributions parallel and perpendicular to the molecular axis, respectively. In contrast with the $(L)$ correlations which are fully isotropic in their $x, y, z$ portions, Eqs. (8) and (9) reflect the inherent cylindrical symmetry of the $(M)$ frame. The short-time expansion of all these correlations are reported in Appendix A.

The details of our MD simulation can be summarized as follows. A number $N=512 \mathrm{HF}$ molecules is enclosed in a cubic box of edge $L$ chosen in such a way that the quantity $\left(N / L^{3}\right) M$ reproduces the correct mass density $1.17 \mathrm{~g} / \mathrm{cm}^{3}$ (at $203 \mathrm{~K}$ ) and $1.015 \mathrm{~g} / \mathrm{cm}^{3}$ (at $273 \mathrm{~K}$ ). The molecules were assumed to interact with the site-site potential discussed in Ref. 6. The dynamics of the particles was determined by integrating the equations of motion with a time step $\delta t$ $=2 \mathrm{fs}$. The assumed rigidity of the molecules was introduced by means of the so-called "constraint method." ${ }^{, 7}$ After equilibration, the molecules have been followed for a MD run of total duration $1.32 \mathrm{~ns}(660000 \delta t)$ at $203 \mathrm{~K}$, and 740 ps $(370000 \delta t)$ at $273 \mathrm{~K}$.

In addition to the standard structural properties, the MD simulation provided values of the Einstein frequency $\Omega_{0}$ and of the diffusion coefficient $D$ (see Table I). Note that the clear discrepancy between the MD and experimental values of $D$ is to be largely ascribed to the simplified potential model adopted in the present simulations, where the dipole moment has been fixed to the value of the isolated molecule (certainly lower than in the liquid phase). Although this limitation can be removed by adopting a polarizable potential model, ${ }^{8}$ in the simulations we have chosen to investigate the
TABLE I. The Einstein frequency $\Omega_{0}$ and the diffusion coefficient $D$ in the two states of HF compared with the corresponding values obtained for the $\mathrm{SPC} / \mathrm{E}$ model of water at $255 \mathrm{~K}$. The experimental values of $D$ are reported in brackets.

\begin{tabular}{lcl}
\hline \hline & $\Omega_{0}\left(\mathrm{ps}^{-1}\right)$ & $D\left(\AA^{2} / \mathrm{ps}\right)$ \\
\hline $\mathrm{HF}(203 \mathrm{~K})$ & 33.5 & $0.31\left(0.127^{\mathrm{a}}\right)$ \\
$\mathrm{F}(273 \mathrm{~K})$ & 30.8 & $1.04\left(0.58^{\mathrm{a}}\right)$ \\
$\mathrm{H}_{2} \mathrm{O}(255 \mathrm{~K})$ & $35.8^{\mathrm{b}}$ & $0.08^{\mathrm{b}}\left(0.05^{\mathrm{c}}\right)$ \\
\hline \hline
\end{tabular}

${ }^{\mathrm{a}} \mathrm{N}$. Karger, T. Vardag, and H.-D. Lüdemann, J. Chem. Phys. 100, 8271 (1994).

${ }^{b}$ D. Bertolini and A. Tani, Phys. Rev. E 56, 4135 (1997).

${ }^{\mathrm{c}} \mathrm{C}$. A. Angell, in Water, A Comprehensive Treatise, edited by (Plenum, New York, 1982), Vol. VII.

simpler nonpolarizable model, which is less demanding from the computational point of view and leaves the features relevant for our study virtually unchanged.

As discussed previously, it is also convenient to introduce a mobile $(M)$ reference frame which follows the dynamics of each molecule. Since for linear molecules like HF the definition of the axes orthogonal to the molecular axis is arbitrary, various artificial ways to overcome the problem have been envisaged. In Ref. 9 the ambiguity was solved by attaching a massless "dummy atom" outside the molecular axis of each molecule. In the present work we ran instead the simulations without any extra atom, introducing axes orthogonal to the instantaneous molecular direction only in the calculation of correlation functions from the stored configurations. This was accomplished by solving numerically the equation of motion for a rigid HF molecule where at time $t$ $=0$ of each set of trajectories a "unit vector" normal to the molecular axis was selected in the direction of the angular momentum. Specifically, for each molecule the dynamics of the unit vector $\mathbf{i}_{\xi}$ orthogonal to the molecular axis was described by

$$
\mathbf{i}_{\xi}(t+\delta t)=\mathbf{i}_{\xi}(t)+\dot{\mathbf{i}}_{\xi}(t) \delta t+(1 / 2) \ddot{\mathbf{i}}_{\xi}(t)(\delta t)^{2}+\cdots,
$$

where $\delta t=2 \mathrm{fs}$ is the time step for integration, and

$$
\dot{\mathbf{i}}_{\xi}(t)=\boldsymbol{\omega}(t) \times \mathbf{i}_{\xi}(t),
$$

where $\boldsymbol{\omega}(t)=\mathbf{i}_{\zeta}(t) \times \dot{\mathbf{i}}_{\zeta}(t)$ is the angular velocity vector. Because of the smallness of $\delta t$ and of the slow reorientation dynamics of the molecules, Eq. (10) can be stopped at the terms linear in $\delta t$. The "truncation error"' in the expansion depends on the relevance of

$\ddot{\mathbf{i}}_{\xi}(t)=(d / d t)\left[\boldsymbol{\omega}(t) \times \mathbf{i}_{\xi}(t)\right]=\dot{\boldsymbol{\omega}}(t) \times \mathbf{i}_{\xi}(t)+\boldsymbol{\omega}(t) \times \dot{\mathbf{i}}_{\xi}(t)$,

where the torque $\mathbf{T}(t)=I \dot{\boldsymbol{\omega}}(t)$ can readily be evaluated. Any small deviation due to the truncated expansion can be corrected by taking the orthogonal projection of the calculated $\mathbf{i}_{\xi}(t)$ onto the rotating molecular axis. To reduce any accumulation of errors, each set of trajectories is further divided into "segments," and the above procedure is carried out independently on each of them. The overall calculation is eventually tested by checking the correct short-time expansions of the various CFs. The additional unit vector $\mathbf{i}_{\eta}(t)$ is simply evaluated by means of

$$
\mathbf{i}_{\eta}(t)=\mathbf{i}_{\zeta}(t) \times \mathbf{i}_{\xi}(t) .
$$




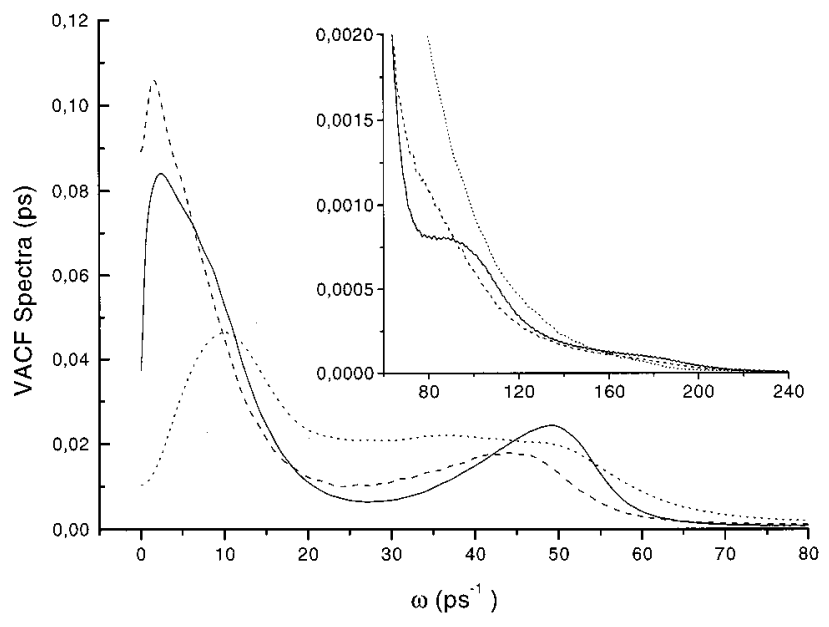

FIG. 3. The VACF frequency spectrum for the three systems of Fig. 1 (same labeling). The high-frequency portion of the spectra is emphasized in the insert.

\section{ANALYSIS OF THE ATOMIC VELOCITY CORRELATIONS AND OF THE ASSOCIATED QUANTITIES}

The presence in the c.o.m. VACF of liquid HF both of wiggles and of a long-lasting tail (see Fig. 1) is reflected in its frequency spectrum $C_{c}^{(L)}(\omega)$, a sort of density of states in a classical liquid. In Fig. 3, the HF data again refer to the two thermodynamic states mentioned in Sec. II. In both the states of $\mathrm{HF}$ one notes qualitative differences with respect to the corresponding spectrum of water; in particular, the much higher value at $\omega=0$ is due to the larger diffusivity of $\mathrm{HF}$ molecules. Even at $203 \mathrm{~K}$, where $D$ is lower, the presence of the VACF tail causes a considerable increase of lowfrequency contributions. Moreover, the initial oscillations of the VACF of HF (basically due to microscopic "cage effects") sharpen the peak at $\omega=45-50 \mathrm{ps}^{-1}$, whereas in water the same physical effect yields a broad shoulder in the same frequency range. Finally, in $\mathrm{HF}$ at $203 \mathrm{~K}$ a closer examination of the high-frequency portion of the VACF spectrum shows an additional feature at $\omega \approx 100 \mathrm{ps}^{-1}$ (see the insert of Fig. 3).

A possible clue of interpretation is provided by the analysis of the atomic VACFs $C_{11}^{(L)}(t), C_{22}^{(L)}(t)$, and $C_{12}^{(L)}(t)$ in HF at 203 and $273 \mathrm{~K}$ (Fig. 4). From the definition of the center-of-mass one deduces that

$$
C_{c}^{(L)}(t)=\frac{r_{m} C_{11}^{(L)}(t)+\left(1 / r_{m}\right) C_{22}^{(L)}(t)+2 C_{12}^{(L)}(t)}{2+r_{m}+\left(1 / r_{m}\right)} .
$$

The spectral contributions to Eq. (14) in different frequency ranges are reported in Figs. 5 and 6, referring to $203 \mathrm{~K}$ and $273 \mathrm{~K}$, respectively. It is apparent that the hydrogenhydrogen contribution $C_{22}^{(L)}(\omega)$ is modest at low and intermediate frequencies, and quite important at high frequencies where the peak around $\omega \approx 110 \mathrm{ps}^{-1}$ can be interpreted as a librational effect naturally emphasized by the small mass of hydrogen. As expected, the fluorine-fluorine spectrum $C_{11}^{(L)}(\omega)$ is basically similar to one of the centers-of-mass, the only difference being a larger amplitude of the highfrequency portion. The mixed contribution $C_{12}^{(L)}(\omega)$ merely "transmits" the dominant spectral features of hydrogen to fluorine (and vice versa); note, however, that at $203 \mathrm{~K}$ the negative contribution of $C_{12}^{(L)}(\omega)$ is not sufficient to cancel any trace of the libration in the dynamics of the center-ofmass, and a remnant of this persists as a broad shoulder.

Further pieces of information are provided by an alternative decomposition which exploits the rigid-motion condition. Using Eq. (6) as an ingredient for the two velocities entering the atomic correlations $C_{\alpha \beta}^{(L)}(t)$ and exploiting the properties of classical statistical averages one obtains

$$
\begin{aligned}
& C_{11}^{(L)}(t)=C_{c}^{(L)}(t)+2 \rho_{1} C_{R T}^{(L)}(t)+\left(\rho_{1}\right)^{2} C_{\omega}^{(L)}(t), \\
& C_{22}^{(L)}(t)=C_{c}^{(L)}(t)-2 \rho_{2} C_{R T}^{(L)}(t)+\left(\rho_{2}\right)^{2} C_{\omega}^{(L)}(t),
\end{aligned}
$$
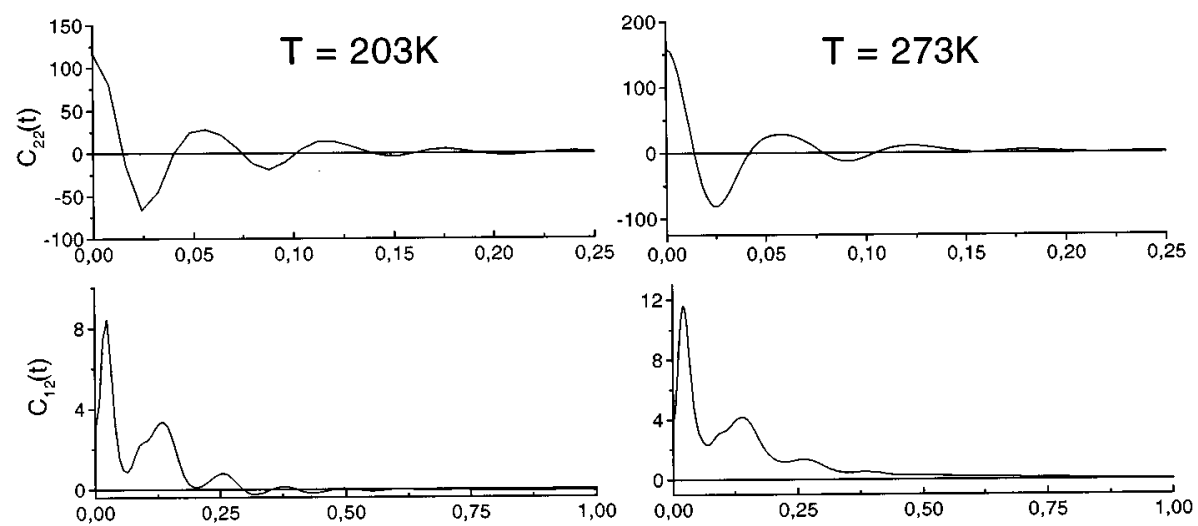

FIG. 4. The three unnormalized atomic VACFs $C_{\alpha \beta}(t)$ in liquid $\mathrm{HF}$ at $203 \mathrm{~K}$ and at $273 \mathrm{~K}$. Labels: $1=$ fluorine, 2 =hydrogen.

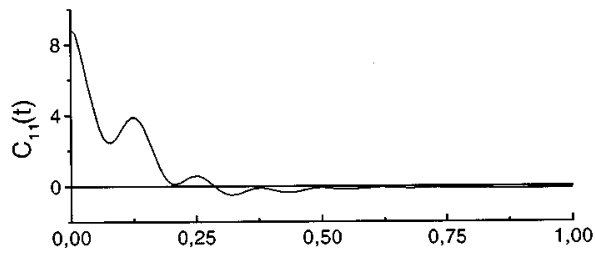

$t(p s)$

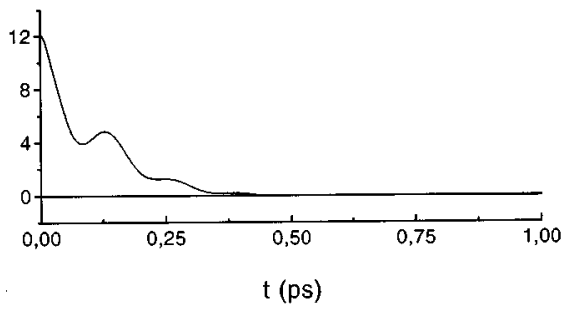




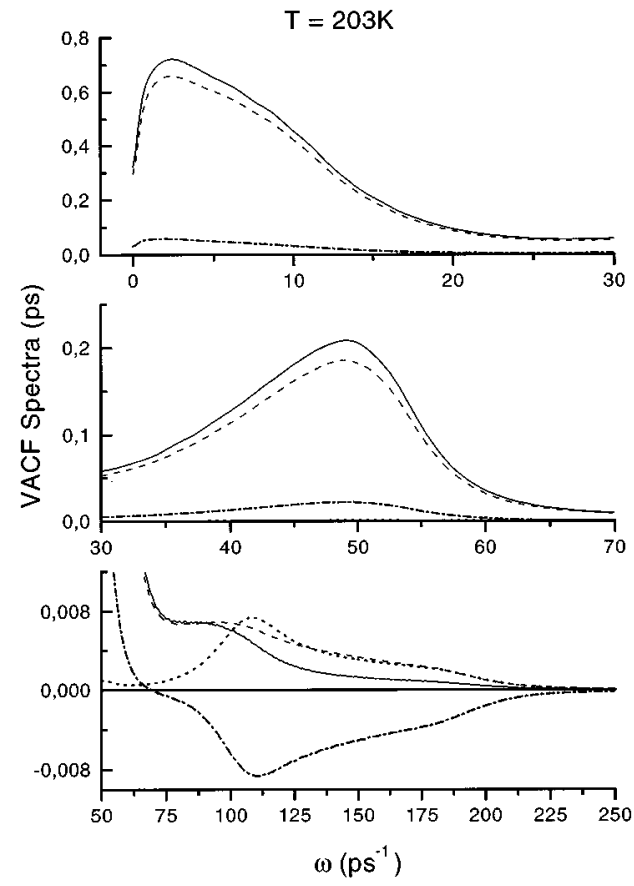

FIG. 5. The spectral weights of the different $C_{\alpha \beta}^{(L)}(\omega)$ with respect to the c.o.m. VACF spectrum $C_{c}^{(L)}(\omega)$ [Fourier transform of Eq. (15)] in HF at $203 \mathrm{~K}$. The data refer to $C_{c}^{(L)}(\omega)$ (full line), $C_{11}^{(L)}(\omega)$ (dashed line), $C_{22}^{(L)}(\omega)$ (dotted line), and $C_{12}^{(L)}(\omega)$ (chain line). On the scale of the graphs at low and intermediate frequencies, the intensity of the hydrogen spectrum $C_{22}^{(L)}(\omega)$ is extremely small.

$$
C_{12}^{(L)}(t)=C_{c}^{(L)}(t)-\left(\rho_{2}-\rho_{1}\right) C_{R T}^{(L)}(t)-\rho_{1} \rho_{2} C_{\omega}^{(L)}(t),
$$

where

$$
C_{R T}^{(L)}(t) \equiv-\left\langle\left[\boldsymbol{\omega}(0) \times \mathbf{i}_{\zeta}(0)\right] \cdot \mathbf{v}^{(c)}(t)\right\rangle,
$$
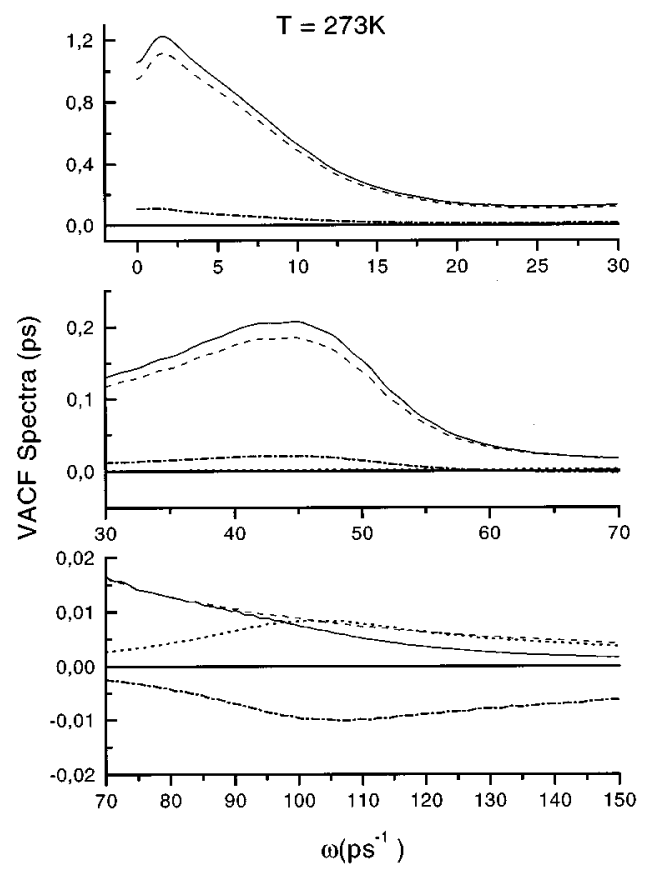

FIG. 6. As in Fig. 5, but at $273 \mathrm{~K}$

$$
C_{\omega}^{(L)}(t) \equiv\left\langle\left[\boldsymbol{\omega}(0) \times \mathbf{i}_{\zeta}(0)\right] \cdot\left[\boldsymbol{\omega}(t) \times \mathbf{i}_{\zeta}(t)\right]\right\rangle .
$$

Assuming that all the $C_{\alpha \beta}^{(L)}(t)(\alpha=1,2)$ are known, system (15) can be solved to find $C_{c}^{(L)}(t), C_{R T}^{(L)}(t)$, and $C_{\omega}^{(L)}(t)$. The result for $C_{c}^{(L)}(t)$ of course reproduces Eq. (14), while

$$
\begin{aligned}
& \rho_{1}\left(1+r_{m}\right)^{2} C_{R T}^{(L)}(t)= r_{m} C_{11}^{(L)}(t)-C_{22}^{(L)}(t) \\
&-\left(r_{m}-1\right) C_{12}^{(L)}(t), \\
&\left(\rho_{1}\right)^{2}\left(1+r_{m}\right)^{2} C_{\omega}^{(L)}(t)=C_{11}^{(L)}(t)+C_{22}^{(L)}(t)-2 C_{12}^{(L)}(t) .
\end{aligned}
$$

It is worthwhile to note that similar algebraic manipulations can be performed starting from the atomic correlations in the molecular frame $C_{\alpha \beta}^{(M)}(t)$, the final results being formally identical to Eqs. (14)-(19) with the superscript $(M)$ replacing everywhere $(L)$. However, in this case the appearance of intrinsically different quantities leads to rather interesting features. Consider, for example, the molecular frame counterparts of the definitions (16) and (17),

$$
C_{R T}^{(M)}(t) \equiv-\sum_{\sigma}\left\langle\left[\boldsymbol{\omega}(0) \times \mathbf{i}_{\zeta}(0)\right] \cdot \mathbf{i}_{\sigma}(0) \mathbf{v}^{(c)}(t) \cdot \mathbf{i}_{\sigma}(t)\right\rangle,
$$

$$
\begin{aligned}
C_{\omega}^{(M)}(t) \equiv & \sum_{\sigma}\left\langle\left\{\left[\boldsymbol{\omega}(0) \times \mathbf{i}_{\zeta}(0)\right] \cdot \mathbf{i}_{\sigma}(0)\right\}\right. \\
& \left.\times\left\{\left[\boldsymbol{\omega}(t) \times \mathbf{i}_{\zeta}(t)\right] \cdot \mathbf{i}_{\sigma}(t)\right\}\right\rangle .
\end{aligned}
$$

Since the angular velocity $\boldsymbol{\omega}(t)$ is always perpendicular to the instantaneous molecular axis, we may set $\boldsymbol{\omega}(t)$ $=\omega_{\xi}(t) \mathbf{i}_{\xi}(t)+\omega_{\eta}(t) \mathbf{i}_{\eta}(t)$ and conclude that

$$
\begin{aligned}
C_{R T}^{(M)}(t) & =-\left\langle\omega_{\eta}(0) \mathbf{v}_{\xi}^{(c)}(t)\right\rangle+\left\langle\omega_{\xi}(0) \mathbf{v}_{\eta}^{(c)}(t)\right\rangle \\
& =2\left\langle\omega_{\xi}(0) \mathbf{v}_{\eta}^{(c)}(t)\right\rangle, \\
C_{\omega}^{(M)}(t) & =\left\langle\omega_{\xi}(0) \omega_{\xi}(t)\right\rangle+\left\langle\omega_{\eta}(0) \omega_{\eta}(t)\right\rangle \\
& =2\left\langle\omega_{\xi}(0) \omega_{\xi}(t)\right\rangle,
\end{aligned}
$$

where in the last steps we have exploited the cylindrical symmetry inherent to the HF molecule. As a result, the quantities $C_{\omega}^{(M)}(t)$ and $C_{R T}^{(M)}(t)$ can be given the usual meaning of angular velocity $\mathrm{CF}$ and of rototranslational coupling in the molecular frame. ${ }^{9}$ However, in the laboratory description an analogous interpretation of $C_{\omega}^{(L)}(t)$ and $C_{R T}^{(L)}(t)$ in terms of basic quantities is no longer valid. First of all, it is easily deduced that

$$
\begin{aligned}
C_{\omega}^{(L)}(t)= & \left\langle[\boldsymbol{\omega}(0) \cdot \boldsymbol{\omega}(t)]\left[\mathbf{i}_{\zeta}(0) \cdot \mathbf{i}_{\zeta}(t)\right]\right\rangle \\
& -\left\langle\left[\boldsymbol{\omega}(0) \cdot \mathbf{i}_{\zeta}(t)\right]\left[\boldsymbol{\omega}(t) \cdot \mathbf{i}_{\zeta}(0)\right]\right\rangle .
\end{aligned}
$$

The presence in Eq. (24) of the unit vectors $\mathbf{i}_{\zeta}(0), \mathbf{i}_{\zeta}(t)$ (namely of the orientational dynamics) makes $C_{\omega}^{(L)}(t)$ different from the usual angular velocity correlation function $\langle\boldsymbol{\omega}(0) \cdot \boldsymbol{\omega}(t)\rangle$. The case of rototranslational coupling is more striking. In fact, the simplest conceivable form of this coupling in the laboratory frame [i.e., $\left.\left\langle\boldsymbol{\omega}(0) \cdot \mathbf{v}^{(c)}(t)\right\rangle\right]$ identically vanishes for diatomic molecules, because of the opposite role that inversion has on the variables $\boldsymbol{\omega}$ and $\mathbf{v}^{(c)}{ }^{9}$ This argument no longer holds for $C_{R T}^{(L)}(t)$, which is certainly nonzero. As shown in the next section, the presence of a 


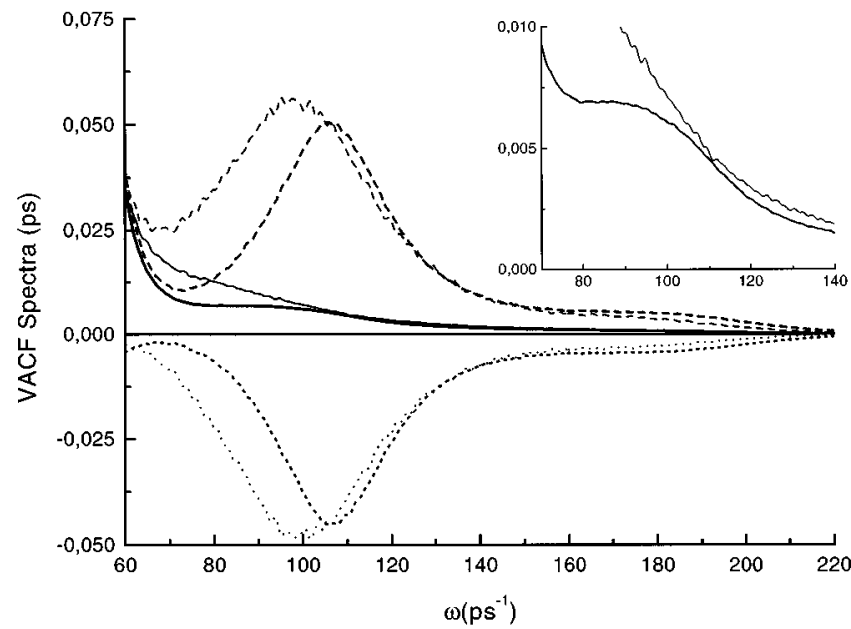

FIG. 7. The spectral role of rototranslational coupling at $203 \mathrm{~K}$ and $273 \mathrm{~K}$ (thick and thin curves respectively), according to the splitting (15c). The plot shows $C_{c}^{(L)}(\omega)$ (full line), $\left(\rho_{2}-\rho_{t}\right) C_{R T}^{(L)}(\omega)$ (dotted line) and $C_{12}^{(L)}(\omega)+\rho_{1} \rho_{2} C_{\omega}^{(L)}(\omega)$ (dashed line). For the two temperatures, the insert magnifies the different spectral behavior of $C_{c}^{(L)}(\omega)$ around $\omega \approx 100 \mathrm{ps}^{-1}$.

nonvanishing $C_{R T}^{(L)}$ is in fact responsible for some of the features apparent in the c.o.m. VACF spectrum.

\section{DISCUSSION}

For clarity, it is convenient to consider the features of the VACF spectrum $C_{c}^{(L)}(\omega)$ in the various frequency ranges.

\section{A. The high frequency part $\left(\omega \approx 100 \mathrm{ps}^{-1}\right)$}

The splitting of the atomic VACFs according to Eqs. (15) permits an alternative interpretation of the features of single-molecule dynamics at high frequencies $\left(\omega \approx 100 \mathrm{ps}^{-1}\right)$. In this range, the "rotational spectrum" $C_{\omega}^{(L)}(\omega)$ is expected (and indeed found) to be qualitatively similar to the hydrogen-hydrogen contribution $C_{22}^{(L)}(\omega)$. Moreover, the Fourier transform of Eq. (15c) can be rearranged to give

$$
\begin{aligned}
C_{c}^{(L)}(\omega)= & \left\{C_{12}^{(L)}(\omega)+\rho_{1} \rho_{2} C_{\omega}^{(L)}(\omega)\right\} \\
& +\left(\rho_{2}-\rho_{1}\right) C_{R T}^{(L)}(\omega) .
\end{aligned}
$$

From the high-frequency contributions of the terms in Eq. (25) one can easily realize the different relevance of the rototranslational coupling $C_{R T}$ at 203 and $273 \mathrm{~K}$ (Fig. 7). In particular, at low temperature the rototranslational coupling in the range $\omega \approx 100 \mathrm{ps}^{-1}$ is such that a remnant of librational effects persists in the left-hand side of Eq. (25). At $273 \mathrm{~K}$, on the other hand, all the features around $\omega \approx 100 \mathrm{ps}^{-1}$ appear to be more smeared out, with the net effect that any remnant of librations in $C_{c}^{(L)}(\omega)$ is hardly visible.

\section{B. The intermediate frequency range $\left(\omega \approx 50 \mathrm{ps}^{-1}\right)$}

As already remarked, the pronounced spectral feature near $45-50 \mathrm{ps}^{-1}$ stems from oscillatory motions of the HF molecule in the "cage" of its neighbors. ${ }^{10}$ This is a wellknown phenomenon more or less present in the VACF spectrum of dense fluids, typically observed at frequencies somewhat higher than the Einstein frequency $\Omega_{0}$. As shown in

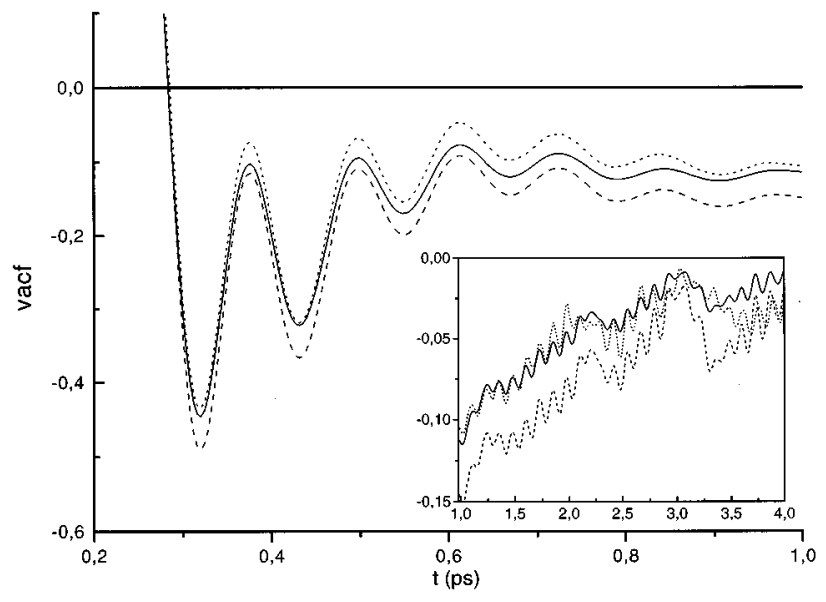

FIG. 8. Intermediate and long time (see insert) behavior of $C_{c}^{(L)}(t)$ (dashed line) vs $C_{c}^{(M)}(t)$ (dotted line) in HF at $203 \mathrm{~K}$. The full line shows $C_{c}^{(M)}(t)$ as obtained from the factorization approximation (26).

Fig. 3, this is also the case for the two states of HF and for $\mathrm{H}_{2} \mathrm{O}$; however, while in water (like in monatomic liquids) the oscillations give rise to a rather broad shoulder, in $\mathrm{HF}$ a well-defined peak is observed. This difference is likely to follow from the peculiar structure of HF, in which the arrangement of the molecules in nearly one-dimensional chains limits any spread of the oscillation frequencies.

\section{Low frequencies [the long time tail of $C_{c}^{(L)}(t)$ ]}

A hint for the origin of the long-lasting behavior of the c.o.m. VACF in $\mathrm{HF}$ at $203 \mathrm{~K}$ is provided by the comparison between $C_{c}^{(L)}(t)$ and $C_{c}^{(M)}(t)$ at sufficiently long times (Fig. $8)$. The main message stands in the fact that $C_{c}^{(M)}(t)$ shows an amplitude of the tail lower than the one of $C_{c}^{(L)}(t)$. An approximate analysis is reported in Appendix B; for the case of interest here, the relevant result can be written as

$$
\begin{aligned}
C_{c}^{(M)}(t) & \approx C_{c}^{(L)}(t) \cdot(1 / 3) \sum_{\sigma}\left\langle\mathbf{i}_{\sigma}(0) \cdot \mathbf{i}_{\sigma}(t)\right\rangle \\
& \equiv C_{c}^{(L)}(t) \cdot(1 / 3)\left[R_{\|}(t)+2 R_{\perp}(t)\right],
\end{aligned}
$$

where the subscript $\|(\perp)$ refers to correlations parallel (perpendicular) to the molecular axis. As shown by Fig. 8, an important part of the slow decay of $C_{c}^{(L)}(t)$ at long times can indeed be traced back to the effect of the long-lasting orientational correlations $R_{\|}(t)$ and $R_{\perp}(t)$, reported in Fig. 9.

Noting that $C_{c}^{(M)}(t)=C_{c, \|}^{(M)}(t)+2 C_{c, \perp}^{(M)}(t)$ and specializing Eq. (26) to components parallel $(\sigma=\zeta)$ or perpendicular $(\sigma=\xi, \eta)$ to the instantaneous molecular axis, one can show that any trace of long-lasting behavior in $C_{c, \|}^{(M)}(t)$ virtually disappears, some long-lasting features still remain in the " 'transverse", contribution $C_{c, \perp}^{(M)}(t)$. The consequent persistence of a (reduced) tail even in $C_{c}^{(M)}(t)$ indicates that the slow decay of the $\mathrm{CF}$ in the laboratory frame cannot totally be ascribed to the slow reorientation of the HF molecules. ${ }^{11}$

A more thorough investigation can be made by abandoning the single-molecule description adopted so far. The simplest way to do this is to consider intermolecular velocity autocorrelation functions of the form 


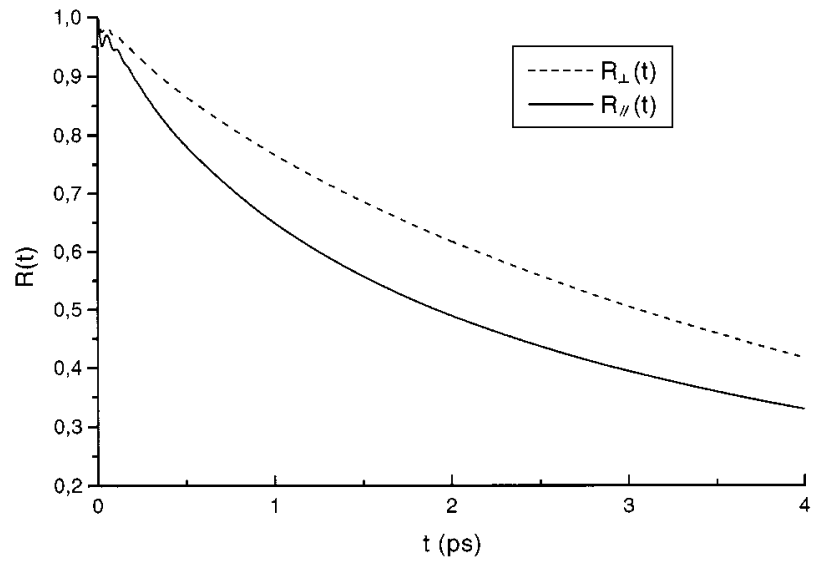

FIG. 9. Orientational correlations parallel and perpendicular to the HF axis at $203 \mathrm{~K}$

$$
\phi^{(i j)}\left(\left|t_{2}-t_{1}\right|\right) \equiv\left\langle\mathbf{v}^{(i)}\left(t_{1}\right) \cdot \sum_{j \neq i}^{\prime} \mathbf{v}^{(i)}\left(t_{2}\right)\right\rangle,
$$

where $\mathbf{v}^{(i)}(t)$ is the c.o.m. velocity of the tagged $i$ th molecule at time $t$, and the apex in $\Sigma$ denotes a summation over the molecules "nearest neighbors" of the tagged one. ${ }^{12}$ In the specific case, two HF molecules are defined to be nearest neighbors if the $\mathrm{F}-\mathrm{F}$ separation is less than the position of the first minimum of the pair distribution function $g_{\mathrm{FF}}(\mathbf{r})$, i.e., $2.66 \AA$ at $203 \mathrm{~K}$ and $2.83 \AA$ at $273 \mathrm{~K}$. The simulation data for the Fourier transform of the "cross" correlation (27) are reported in Fig. 10 together with the single-molecule VACF spectrum. At relatively high frequencies (or short times), the tagged molecule and its neighbors appear to perform a sort of harmonic motion with opposite phases, yielding two nearly opposite bands in the frequency region around $45-50 \mathrm{ps}^{1}$. In this respect, HF is similar to liquid water especially in the supercooled region, ${ }^{13}$ the only difference being that in HF the oscillatory motions are much less damped. On a longer time scale, however, self and cross velocity correlations are seen to behave much in the same way, as clearly shown by the low-frequency region of their spectra. Consequently, in this picture the long-time tail of the VACF appears to reflect a genuine cooperative mechanism.

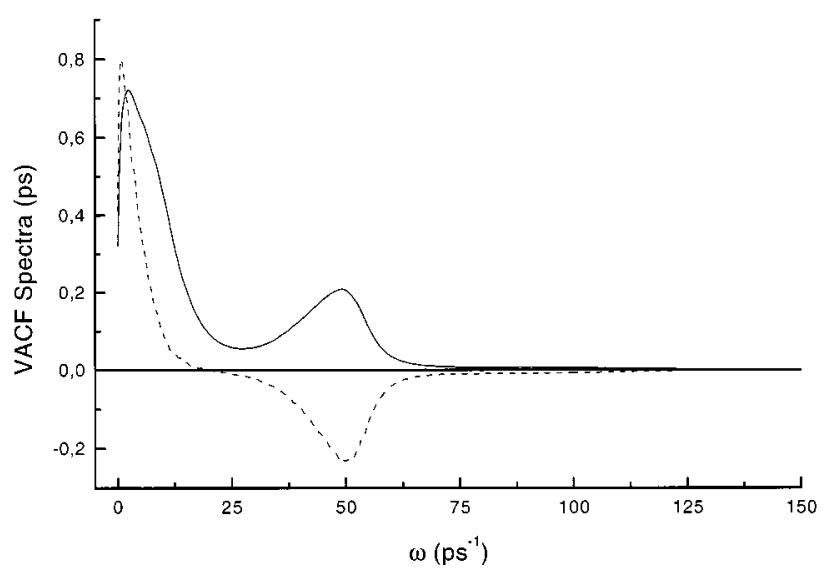

FIG. 10. Self and cross velocity correlation spectra in HF at $203 \mathrm{~K}$ (full and dotted lines, respectively).

\section{CONCLUDING REMARKS}

In this paper we have reported several time correlation functions appropriate to understand the single-molecule dynamics in liquid HF. The basic quantity, the velocity correlation function (VACF) of the molecular center of mass, is found to exhibit at short and intermediate times a number of unusual features particularly when compared with the prototype of hydrogen-bonded fluids, namely liquid water. The structural arrangement of liquid $\mathrm{HF}$ in irregular chains is certainly responsible for most of these peculiarities. In particular, this is the case for the well-defined oscillations detected in the VACF at short and intermediate times, which cause a rather pronounced "cage effect" in the VACF frequency spectrum, both at $T=203 \mathrm{~K}$ (melting point at $\approx 181$ $\mathrm{K}$ ) and at $273 \mathrm{~K}$ (boiling point at $\approx 292 \mathrm{~K}$ ). A more quantitative assessment of the effect by means of a detailed analysis of the VACF memory function is presently in progress. The coupling between translational and rotational motion is shown to play an important role in more subtle highfrequency features present only at $203 \mathrm{~K}$. Conversely, from our MD data it is apparent that rototranslational coupling enhances somewhat the low frequency portion of purely rotational spectrum, "injecting" in the latter a portion of the c.o.m. translational features in this spectral range.

At long times the VACF of HF exhibits a quite slow decay, particularly evident at low temperatures. This longlasting behavior is found to be associated with the slow decay of orientational correlations, ${ }^{14}$ especially in the case of the VACF component parallel to the mobile molecular axis.

\section{APPENDIX A: INITIAL BEHAVIOR OF THE CORRELATION FUNCTIONS}

The values at $t=0$ of the various correlation functions of interest in the laboratory frame are readily calculated. While the translational contributions involve $k_{B} T / M$, the rotational ones can be expressed through the separations $\rho_{1}, \rho_{2}$ $=r_{m} \rho_{1}$ and the moment of inertia $I=m_{1}\left(\rho_{1}\right)^{2}+m_{2}\left(\rho_{2}\right)^{2}$. One finds that

$$
\begin{aligned}
C_{c}^{(L)}(t=0) & =\left\langle\mathbf{v}^{(c)}(0) \cdot \mathbf{v}^{(c)}(0)\right\rangle=3\left(k_{B} T / M\right), \\
C_{11}^{(L)}(t=0) & =\left\langle\mathbf{v}^{(1)}(0) \cdot \mathbf{v}^{(1)}(0)\right\rangle \\
& =3\left(k_{B} T / M\right)+2\left(k_{B} T / I\right)\left(\rho_{1}\right)^{2} \\
& =3\left(k_{B} T / M\right)\left[1+\left(2 / 3 r_{m}\right)\right], \\
C_{22}^{(L)}(t=0) & =\left\langle\mathbf{v}^{(2)}(0) \cdot \mathbf{v}^{(2)}(0)\right\rangle \\
& =3\left(k_{B} T / M\right)+2\left(k_{B} T / I\right)\left(\rho_{2}\right)^{2} \\
& =3\left(k_{B} T / M\right)\left[1+(2 / 3) r_{m}\right], \\
C_{12}^{(L)}(t=0) & =\left\langle\mathbf{v}^{(1)}(0) \cdot \mathbf{v}^{(2)}(0)\right\rangle \\
& =3\left(k_{B} T / M\right)-2\left(k_{B} T / I\right) \rho_{1} \rho_{2}=\left(k_{B} T / M\right),
\end{aligned}
$$

while

$$
C_{R T}^{(L)}(t=0)=-\left\langle\left[\boldsymbol{\omega}(0) \times \mathbf{i}_{\zeta}(0)\right] \cdot \mathbf{v}^{(c)}(0)\right\rangle=0,
$$




$$
\begin{aligned}
C_{\omega}^{(L)}(t=0) & =\left\langle\left|\boldsymbol{\omega}(0) \times \mathbf{i}_{\zeta}(0)\right|^{2}\right\rangle \\
& =\left\langle|\boldsymbol{\omega}(0)|^{2}\right\rangle \\
& =2\left(k_{B} T / I\right) \\
& =2\left(k_{B} T / M\right)\left(l / r_{m} \rho_{1}\right) .
\end{aligned}
$$

After definitions (3) and (4), one deduces that $C_{A B}^{(L)}(t=0)$ $=C_{A B}^{(M)}(t=0)$. Consequently results (A1)-(A6) are valid even in the molecular frame.

The first differences between $C_{A B}^{(L)}(t)$ and $C_{A B}^{(M)}(t)$ arise already at the level of the initial dynamics, namely in the terms of the order $t^{2}$ of the respective short time expansions. Clearly,

$$
C_{A B}^{(L)}(t)=C_{A B}^{(L)}(0)+(1 / 2)\langle\mathbf{A}(0) \cdot \ddot{\mathbf{B}}(0)\rangle t^{2}+\cdots,
$$

where the superscript "dot' denotes a time derivative. On the other hand, by a repeated use of the equation of motion $d \mathbf{i}_{\sigma}(t) / d t=\boldsymbol{\omega}(t) \times \mathbf{i}_{\sigma}(t)$ one finds that

$$
\begin{aligned}
C_{A B}^{(M)}(t)= & C_{A B}^{(M)}(0)+(1 / 2)\langle\mathbf{A}(0) \\
& \left.\cdot\left\{d^{2} / d t^{2}\left[\mathbf{B}(t) \cdot \mathbf{i}_{\sigma}(t)\right]\right\}_{t=0}\right\rangle t^{2}+\cdots \\
= & C_{A B}^{(M)}(0)+(1 / 2)\{\langle\mathbf{A}(0) \cdot \ddot{\mathbf{B}}(0)\rangle \\
& +2\langle\mathbf{A}(0) \cdot[\dot{\mathbf{B}}(0) \times \boldsymbol{\omega}(0)]\rangle \\
& +\langle\mathbf{A}(0) \cdot[\mathbf{B}(0) \times \dot{\boldsymbol{\omega}}(0)]\rangle \\
& +\langle\mathbf{A}(0) \cdot[\mathbf{B}(0) \cdot \boldsymbol{\omega}(0)] \boldsymbol{\omega}(0)\rangle \\
& \left.-\left\langle\mathbf{A}(0) \cdot[\boldsymbol{\omega}(0)]^{2} \mathbf{B}(0)\right\rangle\right\} t^{2}+\cdots .
\end{aligned}
$$

Hence,

$$
\begin{aligned}
C_{A B}^{(M)}(t)= & C_{A B}^{(L)}(t)+(1 / 2)\{2\langle\mathbf{A}(0) \cdot[\dot{\mathbf{B}}(0) \times \boldsymbol{\omega}(0)]\rangle \\
& +\langle\mathbf{A}(0) \cdot[\mathbf{B}(0) \times \dot{\boldsymbol{\omega}}(0)]\rangle \\
& +\langle\mathbf{A}(0) \cdot[\mathbf{B}(0) \cdot \boldsymbol{\omega}(0)] \boldsymbol{\omega}(0)\rangle \\
& \left.-\left\langle\mathbf{A}(0) \cdot[\boldsymbol{\omega}(0)]^{2} \mathbf{B}(0)\right\rangle\right\} t^{2}+O\left(t^{4}\right) .
\end{aligned}
$$

A simple application of Eq. (A9) is the case $\mathbf{A}=\mathbf{B}=\mathbf{v}^{(c)}$, where one finds that

$$
\begin{aligned}
C_{c}^{(M)}(t) / C_{c}^{(M)}(0)= & C_{c}^{(L)}(t) / C_{c}^{(L)}(0) \\
& -(2 / 3)\left(k_{B} T / I\right) t^{2}+O\left(t^{4}\right),
\end{aligned}
$$

a result firstly reported in Ref. 9. In the MD calculations, Eq. (A10) provides a simple check for a consistent introduction of the unit vectors perpendicular to the molecular axis (see Sec. II).

\section{APPENDIX B: CONNECTION BETWEEN THE QUANTITIES IN THE LABORATORY AND IN THE MOLECULAR FRAMES}

A general $\mathrm{CF}$ in the laboratory frame can be written as $(s=x, y, z)$

$$
\begin{aligned}
C_{A B}^{(L)}(t) & =\langle\mathbf{A}(0) \cdot \mathbf{B}(t)\rangle \\
& =\sum_{s}\left\langle A_{s}(0) B_{s}(t)\right\rangle=3\left\langle A_{s}(0) B_{s}(t)\right\rangle .
\end{aligned}
$$

On the other hand, for the corresponding $\mathrm{CF} C_{A B}^{(M)}(t)$ in the molecular frame we may write

$$
\begin{aligned}
C_{A B}^{(M)}(t) & =\sum_{\sigma}\left\langle\mathbf{A}(0) \cdot \mathbf{i}_{\sigma}(0) \mathbf{B}(t) \cdot \mathbf{i}_{\sigma}(t)\right\rangle \\
& =\sum_{s, s^{\prime}}\left\langle A_{s}(0) B_{s^{\prime}}(t) \sum_{\sigma} i_{\sigma, s}(0) i_{\sigma, s^{\prime}}(t)\right\rangle .
\end{aligned}
$$

In the case of diatomic molecules, the rotation matrix $\mathbb{R}(t)$ $=\left(\mathbb{R}_{s, \sigma}(t)\right)=\left(i_{\sigma, s}(t)\right)$ which connects the molecular unit vectors at time $t$ with the laboratory ones can be written as

$$
\begin{aligned}
\mathbb{R}(t) & =\left(\begin{array}{ccc}
\mathbb{R}_{x \xi}(t) & \mathbb{R}_{x \eta}(t) & \mathbb{R}_{x \zeta}(t) \\
\mathbb{R}_{y \xi}(t) & \mathbb{R}_{y \eta}(t) & \mathbb{R}_{y \zeta}(t) \\
\mathbb{R}_{z \xi}(t) & \mathbb{R}_{z \eta}(t) & \mathbb{R}_{z \zeta}(t)
\end{array}\right) \\
& =\left(\begin{array}{ccc}
\cos \phi_{t} \cos \vartheta_{t} & -\sin \phi_{t} & \cos \phi_{t} \sin \vartheta_{t} \\
\sin \phi_{t} \cos \vartheta_{t} & \cos \phi_{t} & \cos \phi_{t} \sin \vartheta_{t} \\
-\sin \vartheta_{t} & 0 & \cos \vartheta_{t}
\end{array}\right),
\end{aligned}
$$

where $\phi_{t}$ and $\vartheta_{t}$ are the polar angles specifying the orientation of the molecular axis at time $t$ with respect to the laboratory axes. In particular, $\vartheta_{t}$ is the angle between the molecular axis at time $t$ and the laboratory $z$-axis. Hence,

$$
C_{A B}^{(M)}(t)=\sum_{s, s^{\prime}}\left\langle A_{s}(0) B_{s^{\prime}}(t) \sum_{\sigma} \mathbb{R}_{s, \sigma}(0) \mathbb{R}_{s^{\prime}, \sigma}(t)\right\rangle .
$$

As it stands Eq. (B4) is not manageable unless we perform some approximation. This is justified when we realize that at sufficiently high density the variables A, B (associated with translational or rotational velocities) are on the whole much faster than the angular variables $\phi$ and $\vartheta$. Consequently, in Eq. (B2) we may approximately factorize the statistical average and write

$$
\begin{aligned}
C_{A B}^{(M)}(t) & \approx \sum_{s, s^{\prime}}\left\langle A_{s}(0) B_{s^{\prime}}(t)\right\rangle \sum_{\sigma}\left\langle i_{\sigma, s}(0) i_{\sigma, s^{\prime}}(t)\right\rangle \\
& =\sum_{s}\left\langle A_{s}(0) B_{s}(t)\right\rangle \sum_{\sigma}\left\langle i_{\sigma, s}(0) i_{\sigma, s}(t)\right\rangle \\
& \equiv C_{A B}^{(L)}(t) \cdot(1 / 3) R(t) \\
& =C_{A B}^{(L)}(t) \cdot(1 / 3)\left[R_{\|}(t)+2 R_{\perp}(t)\right] .
\end{aligned}
$$

Here $\quad R_{\|}(t)=\left\langle\mathbf{i}_{\zeta}(0) \cdot \mathbf{i}_{\zeta}(t)\right\rangle$, while $\quad R_{\perp}(t)=\left\langle\mathbf{i}_{\xi}(0) \cdot \mathbf{i}_{\xi}(t)\right\rangle$ $=\left\langle\mathbf{i}_{\eta}(0) \cdot \mathbf{i}_{\eta}(t)\right\rangle$ because of isotropy in the $M$ frame. Letting $\mathbf{A}=\mathbf{B}=\mathbf{v}^{(c)}$ it is easily verified that at short times the approximation (B5) is consistent with the exact result (A10).

\footnotetext{
${ }^{1}$ See, e.g., A. K. Soper, F. Bruni, and M. A. Ricci, J. Chem. Phys. 106, 247 (1997); V. Röthlisberger and M. Parrinello, ibid. 106, 4658 (1997).

${ }^{2}$ P. Jedlovszky and R. Vallauri, Mol. Phys. 93, 15 (1998).

${ }^{3}$ D. E. O'Reilly, J. Chem. Phys. 49, 5416 (1968).

${ }^{4}$ U. Balucani, G. Ruocco, A. Torcini, and R. Vallauri, Phys. Rev. E 47, 1677 (1993)

${ }^{5}$ D. Bertolini, G. Sutmann, A. Tani, and R. Vallauri, Phys. Rev. Lett. 81, 2080 (1998)

${ }^{6}$ P. Jedlovszky and R. Vallauri, Mol. Phys. 92, 331 (1997).

${ }^{7}$ J. P. Ryckaert, G. Ciccotti, and A. Bellemans, J. Comput. Phys. 23, 327 (1977).

${ }^{8}$ P. Jedlovszky and R. Vallauri, J. Chem. Phys. 107, 10166 (1997).

${ }^{9}$ J. P. Ryckaert, A. Bellemans, and G. Ciccotti, Mol. Phys. 44, 979 (1981).

${ }^{10}$ In a molecular fluid, the same oscillations are usually referred to as
} 
"stretching" intermolecular motions of neighboring molecules.

${ }^{11}$ In this respect, it is worthwhile to compare the low-frequency VACF spectra of $\mathrm{HF}$ with the one of SPC/E water at $255 \mathrm{~K}$ (Fig. 3). In the latter case, owing to the nearly-tetrahedral molecular arrangement, reorientational effects are expected to be less important than in $\mathrm{HF}$. In $\mathrm{H}_{2} \mathrm{O}$, the low-frequency peak at a larger $\omega\left(\approx 11 \mathrm{ps}^{-1}\right)$ has been traditionally attributed to a $\mathrm{O}-\mathrm{O}-\mathrm{O}$ "bending" mode. More recently, contrasting assignments have been proposed for this spectral feature [see, J. Martí, J. A. Padró, and E. Guardia, J. Chem. Phys. 105, 639 (1996); G. Sutmann and R. Vallauri, J. Phys.: Condens. Matter 10, 9231 (1998)].

${ }^{12}$ Strictly speaking, two selection criteria can be adopted according to whether the nearest neighbor condition applies at time $t_{1}$ or $t_{2}$. In our simulations the difference lies below the statistical noise, indicating that on a time scale of a few ps nearest-neighbor molecules remain basically the same. Consequently, the constraint on the summation in Eq. (27) causes no ambiguity.

${ }^{13}$ D. Bertolini, A. Tani, and R. Vallauri, Mol. Phys. 73, 69 (1991).

${ }^{14}$ It is interesting to note that a rather slow decay of orientational correlations has also been reported in liquid methanol [E. Guardia, G. Sesé, and J. A. Padró, J. Mol. Liq. 62, 1 (1994)]. Although this system has a hydrogen bond weaker than HF, the structural arrangement of the molecules in irregular chains is similar. 